\title{
Analytical ultracentrifugation for measuring drug distribution of doxorubicin loaded liposomes in human serum
}

\author{
Dora Mehn • Robin Capomaccio • Sabrina Gioria • \\ Douglas Gilliland • Luigi Calzolai
}

Received: 14 November 2019 / Accepted: 14 April 2020/Published online: 9June 2020

(C) The Author(s) 2020

\begin{abstract}
The potential of analytical ultracentrifugation (AUC) in the analysis of the drug distribution of liposomal doxorubicin formulation (with nominal diameter of $85 \mathrm{~nm}$ ) in the presence of human serum proteins is demonstrated using the absorbance detection function of the instrument. Based on the AUC measurement (and model fitting for molecular mass calculation), we show that in a single experiment, it is possible to measure the relative amounts of the free drug, of the liposomeencapsulated drug, and of the serum protein-bound drug. In addition, the same data provides both the accurate particle size distribution of the liposomal formulation in human serum and information on the protein that binds doxorubicin in the drug-protein fraction (in this case, human serum albumin). Thus, a single experiment (that requires only minimal sample preparation) provides several critical physical-chemical attributes of liposomal drug formulations. This innovative approach will greatly help in the development of improved methods for the challenging problem of characterizing nanomedicine in relevant biological matrices.
\end{abstract}

Keywords Nanomedicine - Analytical ultracentrifugation $\cdot$ AUC $\cdot$ Liposomal drugs

Electronic supplementary material The online version of this article (https://doi.org/10.1007/s11051-020-04843-5) contains supplementary material, which is available to authorized users.

D. Mehn · R. Capomaccio - S. Gioria - D. Gilliland •

L. Calzolai $(\bowtie)$

Joint Research Centre, European Commission, 21027 Ispra, Italy e-mail: luigi.calzolai@ec.europa.eu

\section{Introduction}

Liposomes loaded with different types of drugs are an emerging class of nanotechnology-based innovative therapies, often referred as nanotechnology-enabled pharmaceuticals (NEP). Liposome formulations for the treatment of cancer represent the most abundant class of NEP entering clinical trials in the USA, according to the US Food and Drug Administration (D'Mello et al. 2017). A pegylated liposome encapsulating a cytotoxic drug (doxorubicin) has been one of the first NEP to be approved several years ago for the treatment of breast cancer.

Analytical ultracentrifugation (AUC) was recently demonstrated to be an excellent tool to characterize both the particle size distribution and relative amount of free and encapsulated drug of doxorubicin-loaded liposomes (Mehn et al. 2017). The powerfulness of this technique (developed in the golden age of colloidal chemistry) has long been known and is continuously improved, thanks to the steady development of the freely available software used to fit the sedimentation velocity data with various mathematical models (Schuck et al. 2002). While the $1 \mathrm{~s}-\mathrm{g}^{*}(\mathrm{~s})$ method of SEDFIT has been shown to be very efficient for measuring the size of larger nanoparticles (including liposomes) (Mehn et al. 2017; Wohlleben 2012; Schuck and Rossmanith 2000; Mittal et al. 2010), the real potential of the technique lays in the precise mass analysis of smaller particles and proteins (Schuck 2000; Planken and Colfen 2010; Carney et al. 2011). For example, analytical ultracentrifugation has been successfully applied to the analysis of plasma 
proteins, including the study of protein aggregations in biopharma applications (Liu et al. 2006; Gabrielson et al. 2010; Polyanichko et al. 2017).

Due to their complex nature, the characterization of NEPs is particularly challenging, and several physical-chemical parameters need to be measured for a complete characterization. In particular, the particle size distribution and the amount of free and encapsulated drug represent some of the most important attributes (Halamoda-Kenzaoui et al. 2019). These measurements are not straightforward and require the use and combination of different techniques and careful development of protocols. The particle size distribution can be obtained by different techniques, and amongst those analytical ultracentrifugation has been shown to be a very powerful method (Caputo et al. 2019).

The determination of the free and encapsulated drug uses methods that combine an ultrafiltration step to separate the free drug from liposome-bound fractions, followed by HPLC-UV or LC-MS detection. As described elsewhere, these methods require careful evaluation and case by case development, for example, to ensure that the filtration step does not cause losses of materials (Gioria et al. 2018)

Anticancer liposomal formulations are normally administered by intravenous injection, thus their characterization should be also performed in conditions more similar to the physiological ones, such as, for example, human serum (Bazile 2014). The measurement of the above physic-chemical parameters in such a complex matrix as human serum (that contains a plethora of different proteins and components) is a difficult and time-consuming process (Diou et al. 2015).

Using analytical ultracentrifugation, we show that after incubation of doxorubicin-loaded liposomes with human serum, the drug doxorubicin is present in the three different forms: as free drug, protein bound, and liposome bound. The data allows also estimating the relative distribution of the drug in the three components and the accurate size distribution of the various particles bound to doxorubicin.

\section{Results and discussion}

The AUC instrument can simultaneously measure the absorbance at a specific wavelength in the UV-VIS region and a generic interference signal. Doxorubicin absorbs in the visible region (with a maximum at $490 \mathrm{~nm}$ ), while serum proteins and empty liposomes have negligible absorbance in the visible region (Fig. S1). Thus, setting the UV-VIS detector of AUC at $490 \mathrm{~nm}$ allows the selective monitoring of doxorubicin during the sedimentation process.

Figure 1a shows the absorption-based (at $490 \mathrm{~nm}$, selective for doxorubicin) sedimentation profile of a sample containing a mixture of Dox-NP, human serum proteins, and free doxorubicin. The data indicates the presence of three major components containing doxorubicin (I, II, III).

The first component (I) is fast sedimenting, has a sedimentation coefficient of about $90 \mathrm{~S}$ (Fig. 1b), and indicates the presence of large particles, most probably liposomes. The second component (II) belongs to much smaller particles with a sedimentation coefficient of about $4 \mathrm{~S}$, which could belong to serum proteins. The remaining constant background signal (about 0.25 OD) belongs to the very small, free doxorubicin molecules (component III) with very low sedimentation velocity at 25,000 rpm.

The conversion of the sedimentation profile to the size distribution requires the knowledge of the density of the different particles. For the doxorubicinloaded liposomes (component I), we have previously determined (Mehn et al. 2017) a density of $1.055 \mathrm{~g} /$ $\mathrm{mL}$. Using this density value in the calculation results in a Stokes diameter (mode) of $77 \mathrm{~nm}$ (Fig. 1b, insert), in good agreement with the size specification by the manufacturer of $85 \pm 10 \mathrm{~nm}$ (DLS, hydrodynamic diameter).

Conversion to size in case of component II using the same parameters is not reliable, because the applied ls$\mathrm{g} *(\mathrm{~s})$ model does not consider back diffusion of these small species and because their density is different from that of the liposomes. The fact that the peak appears in the $490 \mathrm{~nm}$ absorbance-based profile indicates that these proteins are bound to doxorubicin, as they sediment together.

A more accurate determination of the size distribution of component II can be obtained by fitting a subset of the sedimentation data with a model that is more appropriate for smaller species with detectable back diffusion. The results of using only the measurement points after the sedimentation of the liposome fraction and fitting them with the continuous c(s) model are shown in Fig. 2. 

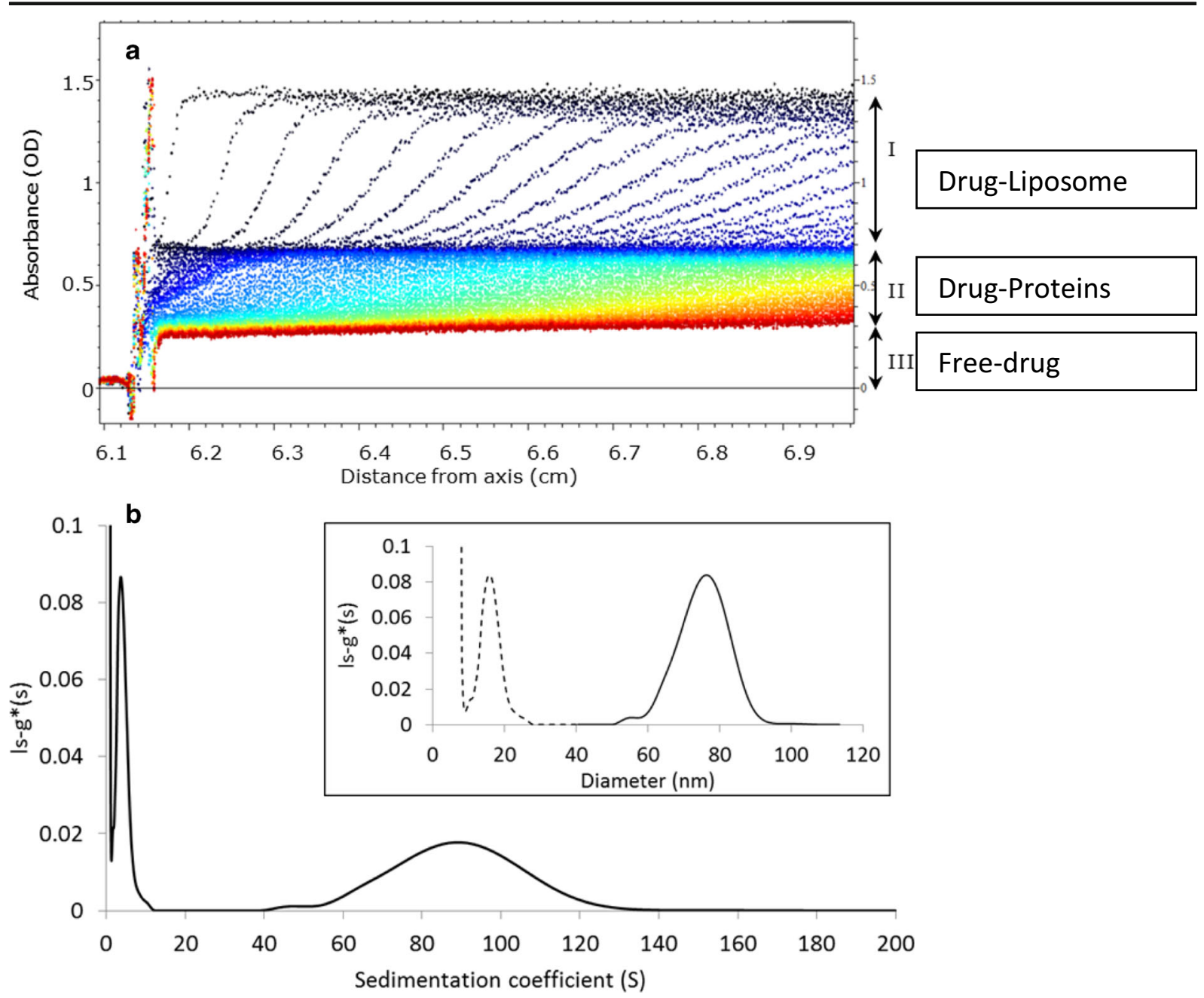

Fig. 1 a Sedimentation profile of the mixture of serum, doxorubicin-loaded liposomes, and doxorubicin measured at $490 \mathrm{~nm}$. Colours represent various time points from first measurement (dark blue) to last (red). Major fractions I, liposomes; II, serum bound doxorubicin; III, free doxorubicin. b Sedimentation

The interference-based molecular mass distribution shows the presence (Fig. 2, black lines) of a major peak at around $67 \mathrm{kDa}$, a second one of around 1/10 lower abundance at $132 \mathrm{kDa}$, and several other ones much less intense at molecular weights higher than $200 \mathrm{kDa}$. The exact values of these higher molecular weights proteins are not reliable because the fit was done with a fixed frictional coefficient ratio which decreases the accuracy of the molecular mass calculation for proteins with different shape (Erickson 2009). These data basically represent the AUC mass distribution profile of proteins from human serum; the most intense peak can be attributed to human serum albumin; it is the most abundant coefficient distribution calculated from the absorption-based measurement. Insert, size distribution of the liposome fraction (solid line). (Dotted line, the size distribution of the protein-bound fraction is inaccurate because of model choice and density)

serum protein (it represents around 50 to $60 \%$ of the protein fraction), and its monomer has a molecular weight of $67 \mathrm{kDa}$. The second strong peak with a maximum at about $132 \mathrm{kDa}$ (slightly above $6 \mathrm{~S}$ ) is most probably a superposition of several different serum proteins, such as HSA dimers, but also alpha- and gammaglobulins, factor $\mathrm{H}$, and even some lipoproteins.

The absorbance-based molecular mass distribution (Fig. 2, red lines) shows the presence of one major component with a molecular mass of $67 \mathrm{kDa}$. This indicates that a good fraction of doxorubicin is found in the serum protein fraction, and in particular, doxorubicin is bound to human serum albumin. This is not 
Fig. 2 Molecular mass distribution of fraction II based on continuous $\mathrm{c}(\mathrm{s})$ model fitting and conversion to molecular mass. Red line (bottom), absorbance based results; Black line (upshifted with 0.02 unit), interference based results. Insert shows the enlarged part of the higher molecular mass region

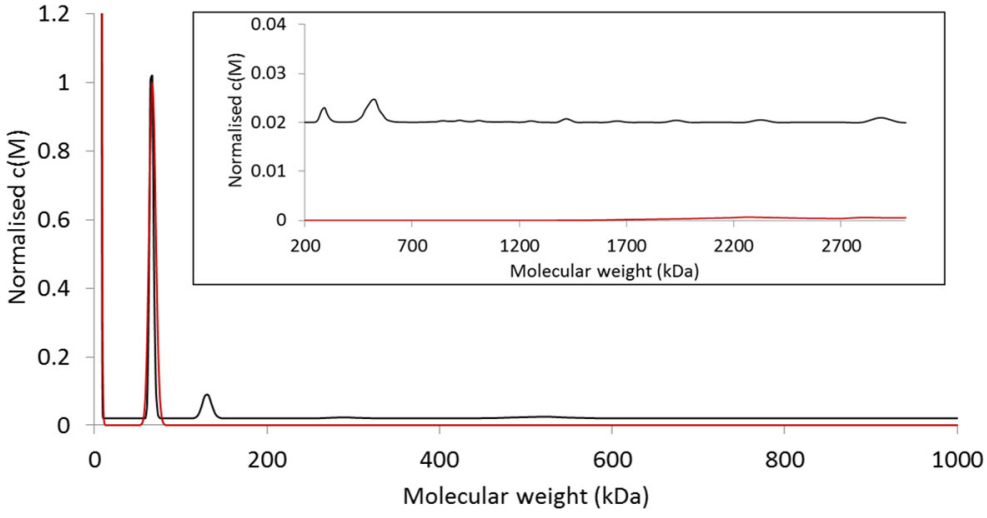

surprising, as HSA is the "classical" drug-transport protein in human serum. The possibility that some smaller fraction of doxorubicin is bound to other proteins cannot be excluded, as the detection limit of this method are quite high, and thus, the combination of low protein concentration with low drug binding could reach below the detection limit of the technique.

To test such possibility, we performed additional measurements where doxorubicin was mixed with either pure HSA or human serum, without the presence of doxorubicin-loaded liposomes. Figure 3 shows both the absorbance-based and the interference-based sedimentation profile of the two samples.

These samples contained particles with a smaller size range than the previous ones. In fact, they do not contain the Dox-NP (size around $70 \mathrm{~nm}$ and sedimentation coefficients bigger than $50 \mathrm{~S}$, see Fig. 2) but only serum components or protein molecules and thus Fig. 2 covers a narrower size range and shows sedimentation distributions at higher resolution.

The two main peaks of the serum sample and HSA appear at the same sedimentation coefficient values in the interference-based distribution (at around $4.2 \mathrm{~S}$ and $6.3 \mathrm{~S}$ ) confirming the contribution of HSA dimers to this signal in serum samples (Fig. 3). The presence of HSA trimers can be also detected in both samples in the 8-9 S sedimentation coefficient range.

The analysis of the absorption-based data results in lower resolution with a second, wider peak positioned between the interference-based peaks of the dimer and trimer. Absorption of these species at $490 \mathrm{~nm}$ might indicate that doxorubicin binds not only to HSA monomers but also to the dimer and trimer forms.

The binding of doxorubicin to HSA dimers and trimers is not observable in the more complex samples containing both serum proteins and liposomes, where a lower resolution data analysis (log spaced grid) is performed only on a subset (component II) of the sedimentation data (Fig. 1a). However, doxorubicin binding to HSA dimers and trimers, in addition to the monomers, has been also confirmed by asymmetric field flow fractionation experiments (Supplementary Fig. S2 online).

In addition to identifying the different forms of doxorubicin in the most complex sample (free drug, drugHSA, drug-liposome), the experiment allows a semiquantitative estimation of the relative concentration of doxorubicin in the three different fractions. In fact the intensities from the absorbance-based sedimentation process measured at the selective frequency of $490 \mathrm{~nm}$ (Fig. 1a) can be directly linked to the concentration of doxorubicin via the Lambert-Beer equation $A=\varepsilon l C$, where $A$ is the measured absorbance, $\varepsilon$ is the molar extinction coefficient, $l$ is the thickness of the cell, and $C$ is concentration. From the experiments, we derived the following absorbance values: $\mathrm{A} 1=0.76 \pm 0.02$, $\mathrm{A} 2=0.41 \pm 0.01$, and $\mathrm{A} 3=0.26 \pm 0.01$, for component I, component II, and component III, respectively.

Knowing that the thickness of the measurement cell (light path in the suspension) is $1 \mathrm{~cm}$, we can estimate the following distribution of doxorubicin amongst the three components:

\section{Component I (drug-liposome): $53.2 \pm 1.3 \%$ \\ Component II (drug-HSA): $28.4 \pm 0.8 \%$ \\ Component III (free drug): $18.5 \pm 0.5 \%$}

This estimation assumes that extinction coefficient $\varepsilon$ is the same for all the doxorubicin forms, but there are evidences that there are some small differences, especially between free and encapsulated doxorubicin.

Initially doxorubicin was added at $50 \%$ as free drug and $50 \%$ as liposome encapsulated. From previous 
Fig. 3 Normalized interferencebased sedimentation coefficient (s) distribution curves of serum (solid grey line) and HSA (solid black line) and absorbance-based sedimentation distribution of serum and HSA (dashed lines) in the presence of doxorubicin

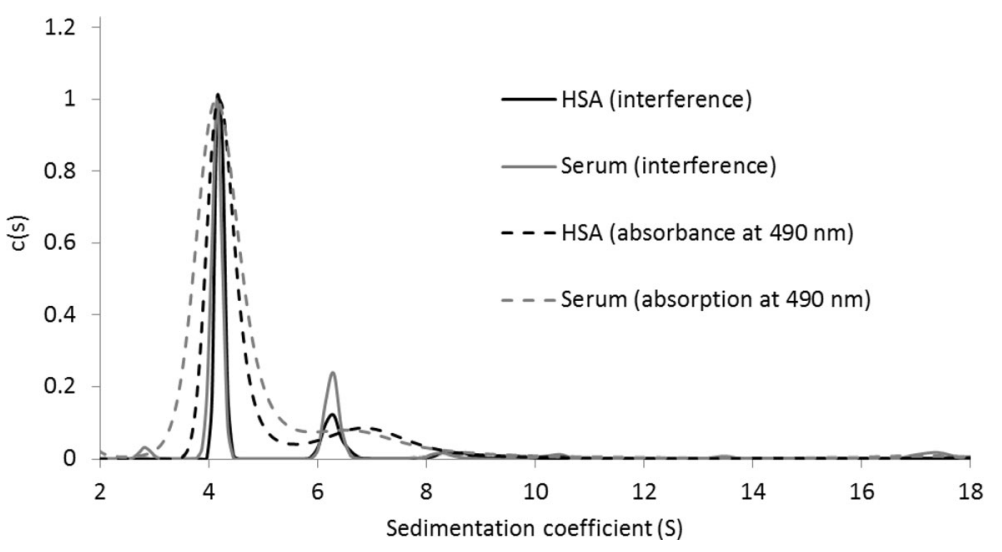

experiments(Mehn et al. 2017), we know that in the Dox-NP sample, there is a maximum of $2.5 \%$ free drug in equilibrium with the loaded (mainly crystalline phase) drug inside the liposomes. The above data indicate that in this system, around $3 / 5$ of the initially added free doxorubicin binds to the protein fraction while about $2 / 5$ stays free in solution. The data also suggest that, at the actually studied concentrations, a small part of the added free doxorubicin binds to the liposomes, most probably contributing to the liposome encapsulated fraction.

\section{Conclusions}

Our results show that AUC with detection at the specific absorption of doxorubicin identifies, in a single experiment with minimal sample preparation, the relative fractions of doxorubicin distributed as free, protein-bound and liposome-bound fraction upon incubation of loaded liposomes with human serum.

In addition to determining the size of the liposomes and to estimating the ratio of the various forms of doxorubicin, the method can also identify the serum protein responsible for the drug binding. In our case, doxorubicin is mainly associated with a protein with a molecular mass of about $67 \mathrm{kDa}$, very likely, human serum albumin (HSA). Our findings (with both AUC and AF4 experiments) also suggest that doxorubicin might be carried also by the dimer (multimer) form of HSA.

It should be possible to apply this method to different types of nanodelivery systems used in nanomedicine, such as organic polymers, dendrimers, and micelles. There are two main possible limitations of this method: the sedimentation process should not perturb the nanosystem, and the drug should have an absorbance in the UV-visible different from the nanocarrier. However, considering the number of nanoformulated products still in the preclinical phase containing active ingredients like anthracyclines, mitoxantrone, or methotrexate, AUC experiments in the presence of serum represent a rapid solution for evaluating drug release and protein binding properties applicable to a large family of nanomedicine candidates.

\section{Methods}

Pegylated liposomes loaded with doxorubicin were purchased from Avanti ${ }^{\circledR}$ Polar Lipids (Lot n. DOXNP5-13, produced by Lipocure Ltd., Jerusalem, Israel). According to the manufacturer's specification, the doxorubicinloaded product contained $2 \mathrm{mg} / \mathrm{mL}$ doxorubicin hydrochloride of which $97.3 \%$ encapsulated in liposomes. Doxorubicin hydrochloride (DoxHCl, European Pharmacopoeia reference standard) and all other chemicals were purchased from Sigma-Aldrich. Phosphatebuffered saline (PBS) solution filtered through a $0.2-\mu \mathrm{m}$ syringe filter (Millipore) before use.

Fresh blood from two healthy volunteers was collected at the JRC medical service previous to an informed consent and in compliance with the Human Tissue Act (2004) and the Human Tissue Authority Code of Practice 1 (Consent). The first $6 \mathrm{~mL}$ collected during phlebotomy was discharged. Z Serum Sep Clot Activator (Cat 454071, Grainer bio-one) has been used for human serum separation. The whole blood from healthy donors drawn into Z Serum Sep Clot Activator was allowed to clot for $40 \mathrm{~min}$, and the serum was obtained by 
centrifugation ( $3500 \mathrm{rpm}, T=4{ }^{\circ} \mathrm{C}$ for $10 \mathrm{~min}$ ). Following centrifugation, the liquid component (serum) fractions of the two donors were mixed together and transferred into sterile 1.5-mL Eppendorf tube using a Pasteur pipette. The samples were maintained at $2-8{ }^{\circ} \mathrm{C}$ while handling. Aliquots of serum of $1 \mathrm{ml}$ were prepared and immediately stored at $-20{ }^{\circ} \mathrm{C}$ for later use.

UV-visible (UV-Vis) spectra of the HSA, control (empty) liposomes and the free drug were recorded in PBS using $0.5-\mathrm{mL}$ quartz cuvettes and a Nicolet Evolution 300 (Thermo) UV-Vis spectrophotometer.

AUC analysis was performed using a Beckman Coulter ProteomeLabTM XL-I analytical ultracentrifuge with an 8 holes rotor, collecting interference and absorbance (490 nm) signals simultaneously in the same run. For AUC analysis of free and encapsulated doxorubicin in the presence of human serum, a $20 \mu \mathrm{L}$ aliquot of the liposome suspension was added to $980 \mu \mathrm{L} 5 \%$ serum containing physiological saline solution and spiked with $40 \mu \mathrm{g} / \mathrm{mL}$ (final concentration) free DoxHCl. Measurement was run at 25,000 rpm rotation speed at $20^{\circ} \mathrm{C}$. Data were fit using the $1 \mathrm{~s}-\mathrm{g}^{*}(\mathrm{~s})$ model in the 1-200 S range, with a logarithmic spaced grid, resolution of 200. Sedimentation data excluding the first 20 scans were also fit using the continuous c(s) model in the 1-60 S range, with a logarithmic spaced grid, resolution of 200, and frictional coefficient ratio of 1.38 .

For AUC analysis of doxorubicin binding on serum proteins and HSA, the measurement was run at a constant rotation speed of $40,000 \mathrm{rpm}$ at $20^{\circ} \mathrm{C}$. The $1 \mathrm{~s}-\mathrm{g} *(\mathrm{~s})$ and continuous c(s) distribution models of the SEDFIT software were applied to fit experimental data to calculate sedimentation coefficient distributions. Sedimentation coefficient distributions were transformed to molecular weight $(\mathrm{M})$ distributions using the "transform $\mathrm{s}$ distribution to M distribution" option of SEDFIT. PBS viscosity and density values were considered to be 0.01022 Poise and $1.0056 \mathrm{~g} / \mathrm{mL}$ at $20^{\circ} \mathrm{C}$. (Brown et al. 2008) Absorbance values measured in the range of 6.2$6.3 \mathrm{~cm}$ distance from the centre of rotation at the first, 6th, and last time point of the measurement series (40 data point/ time point) were used to calculate the absorbance contribution of the various forms of doxorubicin in the liposomes-HSA-doxorubicin hydrochloride mixture.

Online coupled FFF-UV-Vis-MALS-DLS measurements were performed using a Postnova AF4. asymmetric field flow fractionation system connected to a Wyatt's DAWN HELEOS multi-angle light scattering (MALS) detector. HSA solutions (with and without $40 \mu \mathrm{g} / \mathrm{mL}$ DoxHCl) were injected through a $20 \mu \mathrm{L}$ loop into the FFF channel $(350-\mu \mathrm{m}$ spacer, $10 \mathrm{kDa}$ regenerated cellulose membrane). PBS ( $\mathrm{pH} 7.4$ ) was applied as mobile phase at $0.5 \mathrm{~mL} / \mathrm{min}$ flow $3 \mathrm{~mL} / \mathrm{min}$ crossflow and exponentially decreasing crossflow profile and total running times of $30 \mathrm{~min}$. Absorbance of the eluted fractions was monitored at $490 \mathrm{~nm}$ and $280 \mathrm{~nm}$ for the doxorubicin-associated species and total HSA, respectively.

Acknowledgements The authors thank to Rita La Spina for the contribution to the preparation of the serum sample.

\section{Compliance with ethical standards}

Conflict of interest The authors declare that they have no conflict of interest.

Open Access This article is licensed under a Creative Commons Attribution 4.0 International License, which permits use, sharing, adaptation, distribution and reproduction in any medium or format, as long as you give appropriate credit to the original author(s) and the source, provide a link to the Creative Commons licence, and indicate if changes were made. The images or other third party material in this article are included in the article's Creative Commons licence, unless indicated otherwise in a credit line to the material. If material is not included in the article's Creative Commons licence and your intended use is not permitted by statutory regulation or exceeds the permitted use, you will need to obtain permission directly from the copyright holder. To view a copy of this licence, visit http://creativecommons.org/licenses/by/4.0/.

\section{References}

Bazile DV (2014) Nanotechnologies in drug delivery - an industrial perspective. J Drug Deliv Sci Technol 24:12-21 https://doi.org/10.1016/S1773-2247(14)50002-0

Brown PH, Balbo A, Schuck P (2008) Characterizing proteinprotein interactions by sedimentation velocity analytical ultracentrifugation. Curr Protoc Immunol chapter 18:unit 18 15. https://doi.org/10.1002/0471142735.im1815s 81

Caputo F, Clogston J, Calzolai L et al (2019) Measuring particle size distribution of nanoparticle enabled medicinal products, the joint view of EUNCL and NCI-NCL. A step by step approach combining orthogonal measurements with increasing complexity. J Control Release 299. https://doi. org/10.1016/j.jconrel.2019.02.030 
Carney RP, Kim JY, Qian H, Jin R, Mehenni H, Stellacci F, Bakr OM (2011) Determination of nanoparticle size distribution together with density or molecular weight by $2 \mathrm{D}$ analytical ultracentrifugation. Nat Commun 2:335. https://doi. org/10.1038/ncomms 1338

D'Mello SR, Cruz CN, Chen M-L et al (2017) The evolving landscape of drug products containing nanomaterials in the United States. Nat Nanotechnol 12:523-529

Diou O, Greco S, Beltran T, Lairez D, Authelin JR, Bazile D (2015) A method to quantify the affinity of cabazitaxel for PLA-PEG nanoparticles and investigate the influence of the Nano-assembly structure on the drug/particle association. Pharm Res 32:3188-3200. https://doi.org/10.1007/s11095015-1696-0

Erickson HP (2009) Size and shape of protein molecules at the nanometer level determined by sedimentation, gel filtration, and Electron microscopy. Biol Proced Online 11:32-51. https://doi.org/10.1007/s12575-009-9008-x

Gabrielson JP, Arthur KK, Stoner MR, Winn BC, Kendrick BS, Razinkov V, Svitel J, Jiang Y, Voelker PJ, Fernandes CA, Ridgeway R (2010) Precision of protein aggregation measurements by sedimentation velocity analytical ultracentrifugation in biopharmaceutical applications. Anal Biochem 396: 231-241 https://doi.org/10.1016/j.ab.2009.09.036

Gioria S, Caputo F, Urbán P, Maguire CM, Bremer-Hoffmann S, Prina-Mello A, Calzolai L, Mehn D (2018) Are existing standard methods suitable for the evaluation of nanomedicines: some case studies. Nanomedicine 13:539554. https://doi.org/10.2217/nnm-2017-0338

Halamoda-Kenzaoui B, Baconnier S, Bastogne T, Bazile D, Boisseau P, Borchard G, Borgos SE, Calzolai L, Cederbrant K, di Felice G, di Francesco T, Dobrovolskaia MA, Gaspar R, Gracia B, Hackley VA, Leyens L, Liptrott N, Park M, Patri A, Roebben G, Roesslein M, Thürmer R, Urbán P, Zuang V, Bremer-Hoffmann S (2019) Bridging communities in the field of nanomedicine. Regul Toxicol Pharmacol 106:187-196. https://doi.org/10.1016/j. yrtph.2019.04.011

Liu J, Andya JD, Shire SJ (2006) A critical review of analytical ultracentrifugation and field flow fractionation methods for measuring protein aggregation. AAPS J 8:E580-E589. https://doi.org/10.1208/aapsj080367

Mehn D, Iavicoli P, Cabaleiro N, Borgos SE, Caputo F, Geiss O, Calzolai L, Rossi F, Gilliland D (2017) Analytical ultracentrifugation for analysis of doxorubicin loaded liposomes. Int $\mathrm{J}$ Pharm 523:320-326. https://doi.org/10.1016/j. ijpharm.2017.03.046

Mittal V, Volkel A, Colfen H (2010) Analytical ultracentrifugation of model nanoparticles: comparison of different analysis methods. Macromol Biosci 10:754-762. https://doi. org/10.1002/mabi.200900446

Planken KL, Colfen H (2010) Analytical ultracentrifugation of colloids. Nanoscale 2:1849-1869. https://doi.org/10.1039 /c0nr00215a

Polyanichko AM, Mikhailov NV, Romanov NM, Baranova YG, Chikhirzhina EV (2017) Intermolecular interactions in solutions of serum albumin. Cell tissue biol 11:9-15. https://doi. org/10.1134/s1990519x17010084

Schuck P (2000) Size-distribution analysis of macromolecules by sedimentation velocity ultracentrifugation and Lamm equation modeling. Biophys J 78:1606-1619. https://doi. org/10.1016/s0006-3495(00)76713-0

Schuck P, Perugini MA, Gonzales NR, Howlett GJ, Schubert D (2002) Size-distribution analysis of proteins by analytical ultracentrifugation: strategies and application to model systems. Biophys J 82:1096-1111. https://doi.org/10.1016 /s0006-3495(02)75469-6

Schuck P, Rossmanith P (2000) Determination of the sedimentation coefficient distribution by least-squares boundary modeling. Biopolymers 54:328-341. https://doi. org/10.1002/1097-0282(20001015)54:5<328::aid-bip40 $>3.0 . \mathrm{co} ; 2-\mathrm{p}$

Wohlleben W (2012) Validity range of centrifuges for the regulation of nanomaterials: from classification to as-tested coronas. J Nanopart Res 14:1300. https://doi.org/10.1007 /s11051-012-1300-z

Publisher's note Springer Nature remains neutral with regard to jurisdictional claims in published maps and institutional affiliations. 